\title{
Diseño Universal para el Aprendizaje como metodología para la enseñanza de la matemática en la formación de futuros profesores de Educación Especial
}

\author{
Desenho Universal para a Aprendizagem como metodologia para \\ o ensino da matemática na formação dos futuros professores de \\ educação especial
}

\section{Universal Design for Learning as a methodology for teaching} mathematics in the training of special education future teachers

\section{Ximena Gutiérrez-Saldivia'}

Universidad Católica de Temuco, Facultad de Educación, Departamento de Infancia y Educación Básica, Profesora. https://orcid.org/0000-0003-1258-1562

\section{Cecilia Barría Navarro²}

Universidad Católica de Temuco, Facultad de Educación, Departamento de Infancia y Educación Básica, Profesora. https://orcid.org/0000-0002-3556-2615

\section{Danilo Díaz-Levicoy ${ }^{3}$}

Universidad Católica del Maule, Facultad de Ciencias Básicas, Departamento de Matemática, Física y Estadística, Académico. https://orcid.org/0000-0001-8371-7899

Resumen: El tema central del artículo se relaciona con una práctica innovadora en la formación de futuros profesores de Educación Especial, donde se utiliza el Diseño Universal para el Aprendizaje (DUA) como estrategia en la enseñanza de la matemática dentro del curso herramientas de acceso para el aprendizaje de las matemáticas l. Cabe destacar que la implementación del DUA constituye una estrategia para favorecer el aprendizaje de todos los estudiantes y una oportunidad para modelar estrategias de enseñanza diversificadas. El objetivo del trabajo es dar a conocer la implementación del DUA. En este contexto, se analizaron las prácticas de las profesoras del curso y las percepciones de

Máster Universitario en Intervención Psicopedagógica por la Universidad de Granada; doctoranda en Educación en Consorcio por la Universidad Católica de Temuco.

2 Máster en Integración de Personas con Discapacidad por la Universidad de Salamanca; Profesora de Educación Diferencial, Mención Deficiencia Mental por la Universidad Austral de Chile.

3 Doctor en Ciencias de la Educación por la Universidad de Granada; Máster Universitario en Didáctica de la Matemática por la Universidad de Granada. 
las estudiantes, identificándose una metodología de enseñanza tradicional centrada en el profesor, y percepciones negativas de los estudiantes hacia la matemática; luego se seleccionaron e implementaron diferentes estrategias de enseñanza basadas en los tres principios del DUA, sus pautas y puntos de verificación. Finalmente, se puede concluir que la implementación de las estrategias ha permitido una mayor implicancia en el aprendizaje y acercar a los estudiantes a su futuro profesional, aspecto clave en la política educativa de Chile que demanda a las escuelas incorporar el DUA.

Palabras clave: Diseño Universal para el Aprendizaje. Enseñanza de la matemática. Formación de profesores. Educación especial.

Resumo: 0 tema central do artigo relaciona-se com uma prática inovadora na formação dos futuros professores de educação especial, onde utiliza-se o Desenho Universal para a Aprendizagem (DUA) como estratégia no ensino da matemática dentro do curso ferramentas de aceso para o aprendizado das matemáticas l. A implementação do DUA constitui uma estratégia para favorecer a aprendizagem de todos os estudantes e uma oportunidade para modelar estratégias de ensino diversificadas. 0 objetivo do trabalho é divulgar a implementação do DUA. Nesse contexto, foram analisadas as práticas das professoras do curso e as percepções das estudantes, identificando-se uma metodologia de ensino tradicional centrada no professor, e percepções negativas dos estudantes em relação à matemática; depois, diferentes estratégias de ensino baseadas nos três princípios do DUA, suas diretrizes e pontos de verificação, foram selecionados e implementados. Finalmente, conclui-se que a implementação das estratégias permitiu uma maior implicação na aprendizagem e uma aproximação dos estudantes ao seu futuro professional, aspectos fundamentais na poĺtica educativa do Chile, que exige às escolas incorporar o DUA.

Palavras-chave: Desenho Universal para a Aprendizagem. Ensino da matemática. Formação de professores. Educação especial.

Abstract: The central topic of the article is related to an innovative practice in the training of new Special Education teachers, in which Universal Design for Learning (UDL) is used as a strategy for teaching Math in the subject Access Tools for Learning Maths I. The implementation of the UDL is a strategy to facilitate every student's learning, and an opportunity to model diversified teaching strategies. The objective of this work is to reveal the implementation of the UDL. In this context, the teachers' practices in class were analyzed as well as the students' perceptions, identifying a new traditional teaching methodology focused in the teacher, and negative perceptions from students towards Math. Then, different teaching strategies were selected and implemented based on the three principles of the UDL, its guidelines and verification points. Finally, it can be concluded that the implementation of the strategies has permitted a greater involvement in the learning and approaching students to their future careers, which is critical en Chilean educational policies, demanding schools to incorporate UDL.

Keywords: Universal design for learning. Math teaching. Teachers' training. Special education. 


\section{INTRODUCCIÓN}

Este trabajo presenta una práctica innovadora en la formación inicial de profesores de Educación Especial en la Universidad Católica de Temuco (UCT). Esta práctica consistió en la implementación del Diseño Universal para el Aprendizaje (DUA) en el curso herramientas de acceso para el aprendizaje del lenguaje y las matemáticas / de la carrera de Pedagogía en Educación Diferencial. Esta carrera corresponde a un programa de pregrado de la UCT, que tiene como principal propósito formar a profesores competentes para atender y responder a la diversidad, esto desde un enfoque educativo-curricular. La duración de la carrera son 10 semestres académicos, equivalentes a 5 años de estudio. El plan de estudio tiene un total de 300 créditos equivalentes a 8.400 horas, y con un promedio de 60 créditos anuales. En Chile las universidades utilizan un sistema de créditos denominado SCT Chile, que contempla tres tipos de actividades para los estudiantes: horas presenciales, horas mixtas y horas autónomas. Al finalizar el plan de estudio los estudiantes obtienen el título profesional de Profesor de Educación diferencial con Especialidad en Deficiencia Mental y Necesidades Educativas Especiales Transitorias, y el grado académico de Licenciado en Educación. Con respecto al curso herramientas de acceso para el aprendizaje del lenguaje y las matemáticas I, hay que señalar, que se sitúa en el tercer año de la carrera, específicamente en el quinto semestre académico. Este curso es de carácter obligatorio y tiene una carga académica de 10 créditos, correspondiente a 8 horas semanales. Cabe destacar que en este trabajo se optó por utilizar la expresión profesores de educación especial, ya que no en todos los países se utiliza la expresión "educación diferencial" para referirse a los profesores que se desempeñan en el ámbito de la educación especial. En este contexto, nos situamos desde la educación especial como campo de investigación y disciplina científica (GUTIÉRREZ-SALDIVIA, 2014).

El propósito de esta experiencia fue satisfacer las necesidades de aprendizaje de todos los estudiantes de tercer año de la carrera y mejorar los procesos formativos para que estos tengan las mismas oportunidades de lograr los resultados de aprendizajes y competencias profesionales declaradas en la guía de aprendizaje del curso y el perfil de egreso de la carrera. Los participantes fueron 2 profesoras y 80 estudiantes $197 \%$ mujeres y $3 \%$ hombres) que ingresaron a la carrera el año 2015, y que cursaron el año 2017 el curso herramientas de acceso para el aprendizaje del lenguaje y las matemáticas I.

Con respecto a estudios sobre el DUA, señalar que en el contexto internacional existe evidencia científica que el DUA se esta incorporando en la educación formal. Específicamente se han realizado investigaciones en Educación Infantil, Educación Primaria, Educación Secundaria y Educación Superior (ABELL; JUNG; TAYLOR, 2011; BROWDER et al., 2008; 
LIEBER et al., 2008; PARKER; ROBINSON; HANNAFIN, 2008; RAO; WOOK; BRYANT, 2014); aunque existe una mayor cantidad de artículos teóricos que realizan una reflexión crítica sobre la importancia del DUA (MANGIATORDI; SERENELLI, 2013; RAO; WOOK; BRYANT, 2014).

El estudio realizado por Mangiatordi y Serenelli (2013) deja en evidencia que el mayor número de investigaciones se han desarrollado en Estados Unidos, lo que es coherente considerando que este enfoque fue propuesto por el centro de investigación Center for Applied Special Technology (CAST) en Massachusetts. En la actualidad, el DUA se ha convertido en un tópico relevante para las políticas educativas a nivel internacional, pues permiten crear ambientes de enseñanza más inclusivos.

Las investigaciones realizadas por Browder et al. (2008) y Dymond et al. (2006) en Educación Primaria informan que la incorporación del DUA aumenta la participación de estudiantes con discapacidad en las clases y el cumplimiento en los trabajos escolares, además favorece la independencia de los estudiantes en su aprendizaje y mejora las relaciones e interacciones entre estudiantes. En la Educación Secundaria, se destaca el estudio realizado por Kortering, McClannon y Braziel (2008) que analizan la implementación del DUA desde la percepción de los estudiantes, destacando resultados favorables en el aprendizaje de conceptos clave que contribuyeron a mejores rendimientos en exámenes, como también mayores periodos de concentración y aumento en la motivación en el desarrollo de las clases.

En la Educación Superior, la utilización del DUA es valorado positivamente por estudiantes y docentes españoles (ALBA; ZUBILLAGA; SÁNCHEZ, 2015). Parker, Robinson y Hannafin (2008) implementaron el DUA en un curso e-learning, favoreciendo la autonomía en el aprendizaje de los estudiantes y un ambiente de respeto en el aula virtual. Rao y Tanners (2011), en un curso e-learning dirigido a profesores en ejercicio, concluyen que los recursos multimodales basadas en el DUA resultaron útiles para el aprendizaje de los contenidos. Asimismo, Yuval et al. (2004) demuestran una mejora significativa en las actitudes de los estudiantes hacia el aprendizaje y en el rendimiento académico, además manifiestan que es un entorno de aprendizaje beneficioso para estudiantes con discapacidades de aprendizaje. Schelly, Davies y Spooner (2011) afirman que el DUA permite mejorar las tasas de retención y el logro de aprendizaje del alumnado diverso.

En Chile, existe un desarrollo incipiente de estudios sobre el DUA y una politica educativa que promueven la implementación de este enfoque en la Educación Infantil y Primaria como estrategia de atención a la diversidad, mediante el Decreto $N^{\circ} 83$, que aprueba Criterios de Adecuación Curricular (CHILE, 2015). En este contexto, señalar que el Ministerio de Educación comprende el DUA como "una estrategia de respuesta a la diversidad, cuyo fin es maximizar las oportunidades de aprendizaje de todos los estudiantes, considerando la amplia gama de habilidades, estilos de aprendizaje y preferencias." (CHILE, 2015, p. 19). 
Los antecedentes presentados anteriormente y el análisis de la propia práctica pedagógica que realizaron dos profesoras - quienes son autoras de este trabajo- que realizan el curso herramientas de acceso para el aprendizaje del lenguaje y las matemáticas I, motivaron el desarrollo de esta práctica innovadora, la que fue implementada en el año académico 2017.

\section{MARCO DE REFERENCIA DE LA PROPUESTA}

El contexto actual demanda a los sistemas educativos currículums flexibles, para que todos los estudiantes puedan acceder, participar y beneficiarse de la diversidad presentes en las aulas. Sin embargo, aún predominan currículums tradicionales, igual para todos, que establece los mismos objetivos y contenidos. En este contexto, han surgido enfoques que permiten diseñar currículum flexibles y accesibles. Este es el caso del DUA, que surgió en 1984 en Estados Unidos, con la finalidad de apoyar el aprendizaje de todos los estudiantes facilitando el acceso al currículum y los contenidos, mediante tecnologías y recursos didácticos que cumplen funciones específicas (ROSE; MEYER, 2002).

El DUA se define como un marco curricular y didáctico basado en los conocimientos de la neurociencia sobre el aprendizaje (ROSE; MEYER, 2002). Autores como Alba, Zubillaga y Sanchéz (2015), señalan dos hallazgos principales en que se fundamenta el DUA: 1) El aprendizaje se caracteriza por ser modular, distribuido y heterárquico; 2) El aprendizaje se produce de manera diferente en las personas, ya que existe una variabilidad individual en el cerebro (no existen dos cerebros iguales).

En esta misma línea los científicos identifican tres redes cerebrales fundamentales implicadas en un episodio de aprendizaje: afectivas, de reconocimiento y estratégicas (ROSE; MEYER, 2002); y a partir de esto el CAST propone el DUA, que esta organizado en tres principios básicos, que incorporan 9 pautas de trabajo y 31 puntos de verificación, con el propósito de minimizar las barreras del currículo y maximizar las potencialidades de aprendizaje de los estudiantes (MEYER; ROSE; GORDON, 2014). A continuación, se presentan los conceptos clave asociados al DUA.

a) redes afectivas: son las encargadas de evaluar patrones y asignar un significado emocional, generando motivación y compromiso en las situaciones de aprendizaje (ROSE; MEYER, 2002); 
b) redes de reconocimiento: son las que se encargan de la percepción de la información, facilitando la identificación de información y la comprensión de ideas y conceptos (ROSE; MEYER, 2002);

c) redes estratégicas: se encargan de generar y supervisar patrones mentales y motores, permitiendo la planificación y ejecución de las acciones y habilidades (ROSE; MEYER, 2002);

d) principios: son los pilares del DUA que se fundamentan en tres redes neuronales (afectiva, de reconocimiento y estratégica) y que se vinculan a tres aspectos clave del aprendizaje: el por qué del aprendizaje, el qué del aprendizaje y el cómo del aprendizaje (MEYER et al., 2014);

e) pautas: son un conjunto de estrategias que se organizan según los tres principios del DUA para eliminar las barreras presentes en el currículum. Las pautas alinean las redes neuronales con los principios, es decir afectiva con implicancia, reconocimiento con representación, y estratégica con acción (CENTER FOR APPLIED SPECIAL TECHNOLOGY, 2011);

f) puntos de verificación: son estrategias específicas que se organizan según las pautas del DUA para la aplicación en el aula (CENTER FOR APPLIED SPECIAL TECHNOLOGY, 2011).

En em Cuadro 1 se presenta la relación que existe entre las tres redes neuronales, los componentes del diseño curricular (desde el rol del estudiante) y los principios del DUA.

Cuadro 1 - Redes neuronales, principios y pautas del diseño universal para el aprendizaje

\begin{tabular}{|l|l|l|}
\hline \multicolumn{1}{|c|}{ Redes Afectivas } & \multicolumn{1}{c|}{ Redes de reconocimiento } & \multicolumn{1}{c|}{ Redes estratégicas } \\
\hline (Por qué se aprende) & (Qué se aprende) & (Cómo se aprende) \\
\hline $\begin{array}{l}\text { Principio I. Proporcionar múltiples } \\
\text { formas de implicación }\end{array}$ & $\begin{array}{l}\text { Principio II. Proporcionar múltiples } \\
\text { formas de representación }\end{array}$ & $\begin{array}{l}\text { Principio III. Proporcionar múltiples } \\
\text { formas de acción y expresión }\end{array}$ \\
\hline
\end{tabular}

Fuente: adaptado de Gutiérrez-Saldivia et al. (2019).

En el Diagrama 1 se muestran los principios del DUA con sus respectivas pautas para su aplicación en los diseños curriculares (MEYER; ROSE; GORDON, 2014). El principio múltiples formas de implicancia permite que los estudiantes encuentren un incentivo para el aprendizaje y se sientan motivados; el principio múltiples formas de representación permite que los estudiantes perciban y comprendan la información de formas diferentes; y el principio múltiples 
formas de acción y expresión permite a los estudiantes interaccionar con la información y demostrar sus aprendizajes según sus preferencias y capacidades.

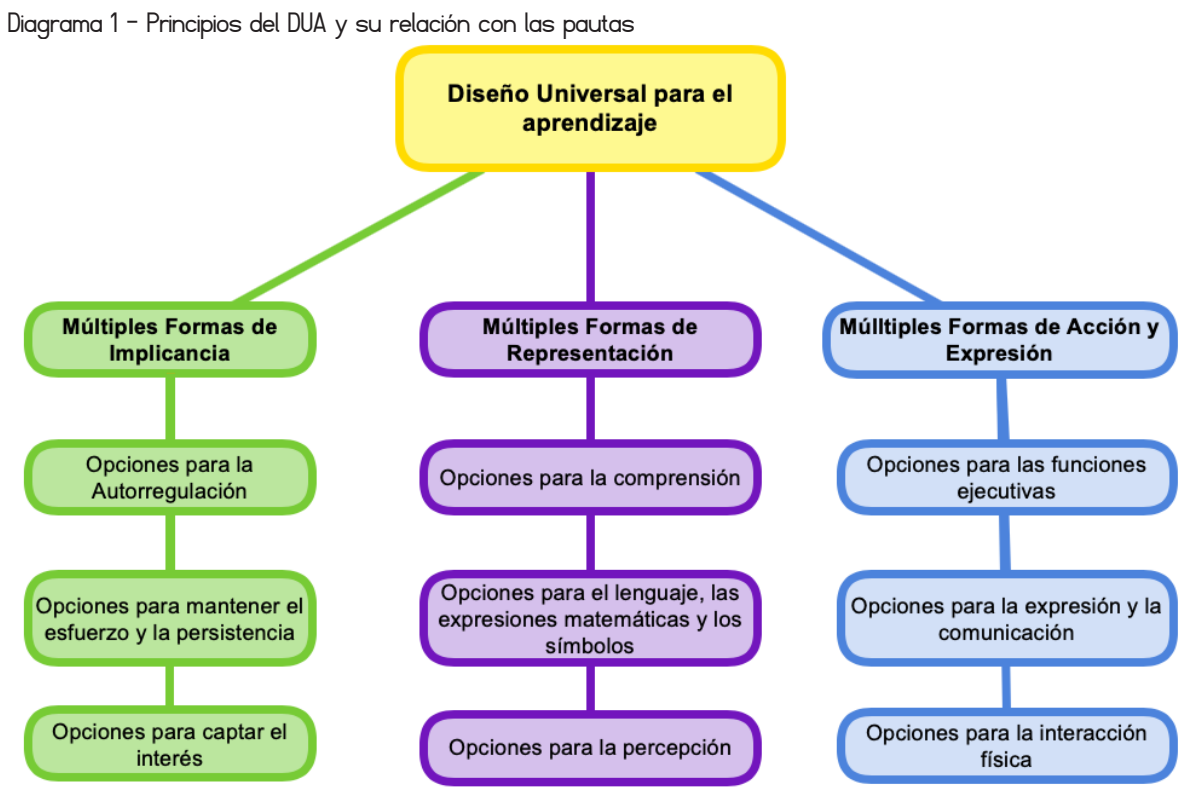

Fuente: elaboración propia a partir de Meyer, Rose y Gordon (2014).

En resumen, el DUA nos aporta elementos teóricos y prácticos para implementar un ambiente de aprendizaje inclusivo en la formación de futuros profesores de educación especial en la Educación Superior.

\section{ANÁLISIS DEL CURSO ANTES DE LA INNOUACIÓN}

El curso herramientas de acceso para el aprendizaje del lenguaje y las matemáticas I considera horas presenciales, autónomas y mixtas. Las horas presenciales se caracterizan por el desarrollo de clases expositivas dialogadas; las horas mixtas están orientadas al trabajo práctico; y las horas autónomas están orientadas al trabajo del estudiante sin la presencia del profesor para la profundización y estudio de las temáticas referidas al curso. Esta estructura se fundamenta en el modelo educativo de la UCT, que se articula en cinco ejes fundamentales (UNIVERSIDAD CATÓLICA DE TEMUCO, 2007). El primero, denominado modelo educativo por competencias involucra como aspecto distintivo, la preocupación por formar 
personas capaces de poner en acción sus conocimientos y recursos personales para la vida personal, el ejercicio profesional y la actuación social. El segundo eje esta asociada al aprendizaje significativo centrado en el estudiante, que esta orientado por principios que regulan tanto los procesos de enseñanza y aprendizaje como los desempeños de estudiantes y académicos, así también las interacciones entre estos, con referencia a las competencias. El tercer y cuarto eje se relacionan con el uso de estrategias institucionales de Tecnologías de la Información y la Comunicación en los procesos de enseñanza y aprendizaje. Por último, el quinto eje formación humanista cristiana exige en sus profesionales claridad en el discernimiento ético, conocimiento disciplinar, habilidad de resolución de conflictos y dominio de competencias.

La principal herramienta con la que cuentan los estudiantes para organizar sus actividades consignadas en el curso es la guía de aprendizaje, que tiene como propósito entregar información general y orientaciones al estudiante sobre el desarrollo de las actividades de aprendizaje y la evaluación del curso (UNIVERSIDAD CATÓLICA DE TEMUCO, 2018). Este instrumento incluye la naturaleza y ponderación de las calificaciones y asistencia exigida, los resultados de aprendizaje que deberán lograr, las competencias del perfil de egreso a las que tributa el curso, los contenidos, métodos y técnicas de enseñanza, el cronograma de actividades y las referencias bibliográficas obligatorias y complementarias. Sin embargo, la guía de aprendizaje no asegura el acceso de todos los estudiantes al aprendizaje, ya que no considera a priori la diversidad presente en el aula. Cabe destacar que el perfil de egreso indica las competencias que el titulado en la UCT debe poseer al término del proceso formativo (UNIVERSIDAD CATÓLICA DE TEMUCO, 2007); entendiéndose las competencias como un "conjunto de conocimientos, destrezas y actitudes capaz de movilizar a una persona, de forma integrada, para que actúe eficazmente ante las demandas de un determinado contexto" (UNIVERSIDAD CATÓLICA DE TEMUCO, 2007, p. 24); mientras que los resultados de aprendizaje corresponden a los "logros específicos que evidencian de forma concreta y tangible la formación y la posesión de una competencia en un determinado nivel." (UCT, 2007, p. 24).

Asimismo, fue importante para la planificación de esta práctica innovadora analizar los resultados de la encuesta aplicada a estudiantes de cohortes anteriores respecto del curso. Los resultados develan que uno de los elementos que más incidencia tiene en el proceso de aprendizaje está relacionado con las barreras para el aprendizaje (85\%) vinculadas con las metodologías de enseñanza implementadas por las profesoras (30\%), las que no siempre facilitan el acceso a los contenidos; también los estudiantes precisan que las estrategias utilizadas por los profesores en ocasiones responden a las características del profesor más que a las diferencias individuales de cada estudiante. Esto también se hace presente en la encuesta de opinión del desempeño docente de los profesores a cargo de este curso, siendo el ítem metodología y preparación la que presenta el promedio más bajo 
en el indicador facilita nuestro aprendizaje con la utilización de diferentes estrategias de enseñanza.

En relación con las estrategias de enseñanza, se han utilizado principalmente métodos expositivos dialogados, acompañados de presentaciones PowerPoint, que incluyen texto e imágenes, y talleres prácticos asociados a juego de roles, análisis de casos, simulación de situaciones de aprendizajes y análisis bibliográfico. En cada sesión los profesores informaban la agenda de la clase con aspectos genéricos de lo que se va a trabajar, se presentaban los aspectos teóricos y se realizaba una actividad práctica. Los recursos utilizados se dejaban a disposición de los estudiantes en la plataforma institucional posterior a las clases, utilizando la plataforma principalmente para la comunicación entre estudiantes y profesores, y como repositorio de materiales. Otro antecedente relevante que se identificó en cursos anteriores es que los estudiantes poseían escasos conocimientos previos sobre el contenido disciplinar matemático.

Los antecedentes presentados anteriormente conllevan a replantearnos como docentes las estrategias de enseñanza que hemos utilizado, y consideramos la necesidad de una reformulación del curso herramientas de acceso para el aprendizaje del lenguaje y las matemáticas / para responder a las diferencias de aprendizajes de los estudiantes y satisfacer efectivamente las necesidades de esta población estudiantil.

En concordancia, se requieren de estrategias inclusivas, ya que el aprendizaje de los saberes de la disciplina requiere de continua práctica y ejecución de los procedimientos y metodologías. En este contexto, seleccionamos el enfoque DUA, ya que permite ser una guía para la apropiación de métodos y formas de enseñar los contenidos del curso, ya que ofrece estrategias para proporcionar múltiples formas de implicación en el aprendizaje, múltiples formas de representación de la información y múltiples formas de acción y expresión.

\section{DESCRIPCIÓN DE LA EXPERIENCIA}

La práctica innovadora tuvo como motivación el interés de las profesoras del curso herramientas de acceso para el aprendizaje del lenguaje y las matemáticas I por mejorar sus prácticas pedagógicas, a fin de favorecer que todos los estudiantes logren los resultados de aprendizaje. En este contexto el equipo de profesoras se planteó como objetivo desarrollar un ambiente de aprendizaje inclusivo mediante la aplicación de estrategias basadas en el DUA para contribuir al logro de los resultados de aprendizaje en un aula diversa. En este contexto, se incorporaron estrategias de enseñanza basadas en los tres principios del DUA: a) múltiples 
opciones para la implicancia; b) múltiples opciones para la representación de la información; y c) múltiples opciones para la expresión y comunicación.

El diseño e implementación de la propuesta de innovación consideró el desarrollo de las siguientes actividades: a) Análisis de la guía de aprendizaje del curso en relación a los métodos y estrategias de enseñanza; b) Selección de estrategias de enseñanza y aprendizaje que respondan a los tres principios del DUA; c) Construcción de una matriz de análisis de estrategias de enseñanza y aprendizaje seleccionados en coherencia con los principios del DUA, sus pautas y puntos de verificación; d) Re-diseño de la guía de aprendizaje del curso; e) Construcción de instrumentos de recogida de datos para la valoración de las estrategias por parte de los estudiantes; f) Implementación de las estrategias en el curso; g) Aplicación de los instrumentos de valoración; y h) Análisis de los datos.

En este trabajo se profundiza en las estrategias implementadas, las que fueron elegidas según el criterio de selección, es decir que respondieran al menos a un principio DUA, con el propósito de ofrecer a los estudiantes múltiples formas de implicancia, múltiples formas de representación de la información, y múltiples formas de acción y expresión, para el aprendizaje de los contenidos didácticos y disciplinares de la matemática. Las estrategias seleccionadas fueron: 1) ruta de trabajo clase a clase; 2) material preparatorio; 3) material didáctico físico-manipulable; 4) material audiovisual; 5) toma de apuntes; 6) foro virtual y wiki. Cada estrategia tributa a los principios del DUA, pautas y puntos de verificación, promoviendo la activación de las redes neuronales afectivas, de reconocimiento y planificación.

\subsection{RUTA DE TRABAJO}

Con respecto a la ruta de trabajo clase a clase señalar que es una planificación de cada clase dirigida a los estudiantes. Esta incluye información sobre la fecha, número de sesión, objetivo de la clase y las actividades correspondientes a las horas presenciales, mixtas y autónomas, que se denominan en la ruta como actividades antes de la clase, durante la clase, y después de la clase. Esta ruta de trabajo era enviada a los estudiantes con al menos 48 horas de anticipación a la clase presencial mediante la plataforma institucional. Desde el enfoque DUA, se potencia la activación de las redes neuronales afectivas, de reconocimiento y de planificación. Específicamente se han seleccionado los tres principios DUA y las pautas para promover el esfuerzo y la persistencia, la comprensión y las funciones ejecutivas. En el Cuadro 2 se especifican los puntos de verificación asociados a la estrategia. 
Cuadro 2 - Matriz de especificación DUA de la estrategia ruta de trabajo clase a clase

\begin{tabular}{|l|l|}
\hline Principio DUA implicado & \multicolumn{1}{c|}{ Pauta y punto de verificación DUA implicados } \\
\hline $\begin{array}{l}\text { Proporcionar múltiples } \\
\text { formas de implicación }\end{array}$ & $\begin{array}{l}\text { Pauta: Proporcionar opciones para mantener el esfuerzo y la persistencia } \\
\text { Punto de verificación: Resaltar la relevancia de las metas y los objetivos } \\
\text { Pauta: Proporcionar opciones para captar el interés } \\
\text { Punto de verificación: Minimizar la sensación de inseguridad y las distracciones }\end{array}$ \\
\hline $\begin{array}{l}\text { Proporcionar múltiples } \\
\text { formas de represen- } \\
\text { tación }\end{array}$ & $\begin{array}{l}\text { Pauta: Proporcionar opciones para la comprensión } \\
\text { Punto de verificación: Guiar el procesamiento de la información, la visualización y la } \\
\text { manipulación }\end{array}$ \\
\hline $\begin{array}{l}\text { Proporcionar múltiples } \\
\text { formas de acción y } \\
\text { expresión }\end{array}$ & $\begin{array}{l}\text { Pauta: Proporcionar opciones para las funciones ejecutivas. } \\
\text { Punto de verificación: Apoyar la planificación y el desarrollo de estrategias } \\
\text { Punto de verificación: Aumentar la capacidad para hacer un seguimiento de los } \\
\text { avances }\end{array}$ \\
\hline
\end{tabular}

Fuente: Adaptado de Barría, Gutiérrez-Saldivia y Tapia (en prensa).

\subsection{MATERIAL PREPARATORIO}

El material preparatorio son recursos -tales como presentaciones PowerPoint y lecturas- que los estudiantes tenían acceso antes de la clase presencial mediante la plataforma virtual. El proporcionar material antes de la clase a los estudiantes tenía como propósito que contaran con un conocimiento previo sobre los contenidos que se abordarían en las horas presenciales para potenciar la comprensión y la participación activa en el aula. Desde el DUA se ha seleccionado el principio múltiples formas de representación y la pauta opciones para la comprensión. En el Cuadro 3 se muestra el punto de verificación asociado a la estrategia.

Cuadro 3 - Matriz de especificación DUA de la estrategia material preparatorio

\begin{tabular}{|l|l|}
\hline \multicolumn{1}{|c|}{ Principio DUA implicado } & \multicolumn{1}{c|}{ Pauta y punto de verificación DUA implicados } \\
\hline $\begin{array}{l}\text { Proporcionar múltiples formas de } \\
\text { representación }\end{array}$ & $\begin{array}{l}\text { Pauta: Proporcionar opciones para la comprensión } \\
\text { Punto de verificación: Activar o sustituir los conocimientos previos }\end{array}$ \\
\hline
\end{tabular}

Fuente: Adaptado de Barría, Gutiérrez-Saldivia y Tapia (sometido) 


\subsection{MATERIAL DIDÁCTICO FÍSICO-MANIPULABLE}

Otra estrategia es el material didáctico físico-manipulable, que corresponden a recursos didácticos estructurados y no estructurados para la enseñanza de la matemática. Entre ellos destacamos los bloques multibase, tablero posicional, tabla de seguín, caja mackinder, tubos conectables, contadores, listones rojos y azules, franjas numéricas, balanza numérica y caja fraccionaria. Cabe destacar que el material didáctico se usó en el aula para que los estudiantes aprendieran el contenido disciplinar y didáctico. Con respecto al DUA señalar que el uso de materiales físicos-manipulables favorece la activación de las redes neuronales de representación y estratégicas. Específicamente se han seleccionado los principios múltiples formas de representación, y múltiples formas de acción y expresión, y las pautas opciones para el lenguaje, las expresiones matemáticas y los símbolos, opciones para la percepción y opciones para la expresión y comunicación. Ver en el Cuadro 4 los puntos de verificación asociados a la estrategia material didáctico físico-manipulable.

Cuadro 4 - Matriz de especificación DUA de la estrategia material didáctico físico-manipulable

\begin{tabular}{|l|l|}
\hline \multicolumn{1}{|c|}{ Principio DUA implicado } & \multicolumn{1}{c|}{ Pauta y punto de verificación DUA implicados } \\
\hline $\begin{array}{l}\text { Proporcionar múltiples } \\
\text { formas de represen- } \\
\text { tación }\end{array}$ & $\begin{array}{l}\text { Pauta: Proporcionar opciones para el lenguaje, las expresiones matemáticas y los } \\
\text { símbolos } \\
\text { Punto de verificación: llustrar la información a través de múltiples medios } \\
\text { Pauta: Proporcionar opciones para la percepción } \\
\text { Punto de verificación: Ofrecer alternativas para la información auditiva } \\
\text { Punto de verificación: Ofrecer alternativas para la información visual }\end{array}$ \\
\hline $\begin{array}{l}\text { Proporcionar múltiples } \\
\text { formas de acción y } \\
\text { expresión }\end{array}$ & $\begin{array}{l}\text { Pauta: Proporcionar opciones para la expresión y la comunicación } \\
\text { Punto de verificación: Usar múltiples herramientas para la construcción y la } \\
\text { composición }\end{array}$ \\
\hline
\end{tabular}

Fuente: adaptado de Barría, Gutiérrez-Saldivia y Tapia (en prensa).

\subsection{MATERIAL AUDIOUISUAL}

El material audiovisual que se utilizó corresponden a videos de clases reales de matemática en aulas chilenas de Educación Primaria, que incluyen subtítulos en castellano y audio. Se accedió a este material a través del libro matemática en aula: docentes en acción texto con recursos multimedia- elaborado por el Centro de Modelamiento Matemático (2016) de la Universidad de Chile. Su elección se debió a que permite fortalecer la formación de futuros profesores mediante la reflexión y análisis de experiencias de enseñanza de la matemática. 
Desde el punto de vista del DUA, estos vídeos responden al principio múltiples formas de representación, y las pautas opciones para el lenguaje, las expresiones matemáticas y los símbolos, y opciones para la percepción. En el Cuadro 5 se especifican los puntos de verificación que se asocian a la estrategia.

Cuadro 5 - Matriz de especificación DUA de la estrategia material audiovisual

\begin{tabular}{|l|l|}
\hline \multicolumn{1}{|c|}{ Principio DUA implicado } & \multicolumn{1}{c|}{ Pauta y punto de verificación DUA implicados } \\
\hline & $\begin{array}{l}\text { Pauta: Proporcionar opciones para el lenguaje, las expresiones matemáticas y los } \\
\text { símbolos }\end{array}$ \\
$\begin{array}{l}\text { Proporcionar múltiples } \\
\text { formas de represen- } \\
\text { tación }\end{array}$ & $\begin{array}{l}\text { Punto de verificación: llustrar la información a través de múltiples medios } \\
\text { Pauta: Proporcionar opciones para la percepción } \\
\text { Punto de verificación: Ofrecer alternativas para la información auditiva } \\
\end{array}$ \\
\hline
\end{tabular}

Fuente: adaptado de Barría, Gutiérrez-Saldivia y Tapia (en prensa).

\subsection{TOMA DE APUNTES}

Esta estrategia consistió en que un grupo de estudiantes tomaba apuntes de los contenidos abordados en cada clase -siendo grupos distintos cada semana- utilizando formas de expresión y comunicación que más les acomodaban. Los apuntes que se generaban eran compartidas posterior a las clases con sus pares en la plataforma para fomentar la colaboración. Así, desde el DUA el propósito era ofrecer múltiples formas de implicancia y múltiples opciones de acción y expresión, a través de las pautas opciones para mantener el esfuerzo y la persistencia, opciones para la expresión y comunicación, y opciones para las funciones ejecutivas. En el Cuadro 6 se informan los puntos de verificación asociados a la toma de apuntes.

Cuadro 6 - Matriz de especificación DUA de la estrategia toma de apuntes

\begin{tabular}{|l|l|}
\hline \multicolumn{1}{|c|}{ Principio DUA implicado } & \multicolumn{1}{c|}{ Pauta y punto de verificación DUA implicados } \\
\hline $\begin{array}{l}\text { Proporcionar múltiples formas } \\
\text { de implicancia }\end{array}$ & $\begin{array}{l}\text { Pauta: Proporcionar opciones para mantener el esfuerzo y persistencia } \\
\text { Punto: Fomentar la colaboración y la comunidad }\end{array}$ \\
\hline $\begin{array}{l}\text { Proporcionar múltiples formas } \\
\text { de acción y expresión }\end{array}$ & $\begin{array}{l}\text { Pauta: Proporcionar opciones para la expresión y comunicación } \\
\text { Punto de verificación: Usar múltiples medios de comunicación }\end{array}$ \\
\hline
\end{tabular}




\begin{tabular}{|l|l|}
\hline \multicolumn{1}{|c|}{ Principio DUA implicado } & \multicolumn{1}{c|}{ Pauta y punto de verificación DUA implicados } \\
\hline $\begin{array}{l}\text { Proporcionar múltiples formas } \\
\text { de acción y expresión }\end{array}$ & $\begin{array}{l}\text { Pauta: Proporcionar opciones para las funciones ejecutivas } \\
\text { Punto de verificación: Facilitar la gestión de información y de recursos }\end{array}$ \\
\hline
\end{tabular}

Fuente: adaptado de Barría, Gutiérrez-Saldivia y Tapia (en prensa).

\subsection{FORO UIRTUAL Y WIKI}

El foro virtual y wiki son herramientas de la plataforma institucional Moodle e-learning. El foro tiene como propósito que los estudiantes puedan plantear sus ideas sobre un tema y discutir sobre ello en base a argumentos. Con respecto a la wiki señalar que es un recurso que permite que los estudiantes puedan diseñar (como también editar) una página web sobre un tema o problemática de manera colaborativa. Tanto el foro virtual como la wiki fueron utilizados en el curso de manera intencionada, siendo los docentes quienes planificaban las actividades asociadas a ambas estrategias. Desde el DUA estas estrategias buscan proporcionar múltiples formas de implicancia y múltiples formas de acción y expresión en los estudiantes, mediante las pautas opciones para mantener el esfuerzo y la persistencia, y opciones para la expresión y comunicación. El Cuadro 7 informa los puntos de verificación asociados al foro y wiki.

Cuadro 7 - Matriz de especificación DUA de la estrategia foro y wiki

\begin{tabular}{|l|l|}
\hline \multicolumn{1}{|c|}{ Principio DUA implicado } & \multicolumn{1}{c|}{ Pauta y punto de verificación DUA implicados } \\
\hline $\begin{array}{l}\text { Proporcionar múltiples formas } \\
\text { de implicancia }\end{array}$ & $\begin{array}{l}\text { Pauta: Proporcionar opciones para mantener el esfuerzo y persistencia } \\
\text { Punto: Fomentar la colaboración y la comunidad }\end{array}$ \\
\hline $\begin{array}{l}\text { Proporcionar múltiples formas } \\
\text { de acción y expresión }\end{array}$ & $\begin{array}{l}\text { Pauta: Proporcionar opciones para la expresión y comunicación } \\
\text { Punto de verificación: Usar múltiples medios de comunicación }\end{array}$ \\
\hline
\end{tabular}

Fuente: adaptado de Gutiérrez-Saldivia, Barría y Tapia (en prensa).

\section{CONSIDERACIONES FINALES}

A partir de la implementación de las estrategias basadas en el DUA en el curso herramientas de acceso para el aprendizaje del lenguaje y las matemáticas I se han identificado las estrategias más valoradas y menos valoradas por los estudiantes. Para esto se utilizó una escala de valoración y cuestionario de preguntas abiertas (GUTIÉRREZ-SALDIVIA; BARRÍA; TAPÍA, en prensa). 
De la práctica innovadora destacamos que los estudiantes valoraron positivamente el material preparatorio y recursos didácticos físicos-manipulables. En este sentido, señalar que el material preparatorio permitió reducir sentimientos negativos de los estudiantes hacia las matemáticas y sensaciones de inseguridad presentes al inicio del curso. En la enseñanza de las matemáticas es importante considerar las emociones y sentimientos de las estudiantes, ya que pueden determinan el éxito o el fracaso (FLORES et al., 2013). En relación a esto, es fundamental que los profesores utilicen estrategias que favorezcan la activación de redes neuronales afectivas, ya que minimizan sensaciones de inseguridad y favorecen la autorregulación de los estudiantes (MEYER; ROSE; GORDON, 2014). Con respecto al material didáctico físico-manipulativo, destacar que fue clave para lograr la comprensión y aprendizaje de los contenidos matemáticos por parte de los futuros profesores, contribuyendo a una mayor motivación e implicancia de los estudiantes en las clases, siendo un mediador en el proceso, y no solo un recurso.

Por otro lado, se han identificado una menor valoración de aquellas estrategias que involucran el trabajo autónomo de los estudiantes, como por ejemplo la toma de apuntes, wiki y foro virtual. Esto es coincidente con la investigación realizada por Dean, Lee-Post y Hapke (2016), quienes informan que los participantes de su estudio tuvieron mayores preferencias por estrategias que incluían contenidos generados por los profesores y bajas preferencias por estrategias para el trabajo autónomo. Esto, podría deberse como consecuencia del modelo de educación bancaria que ha predominado en la sociedad, donde el docente es el único poseedor de conocimientos y los estudiantes son objetos que oyen (FREIRE, 2002).

En cuanto a la labor docente, permitió avanzar desde un enfoque de enseñanza más tradicional centrado en el profesor, a uno más inclusivo en el que los estudiantes asumieron un rol más activo en las clases presenciales. Además, fomentó la colaboración entre docentes, ya que la implementación de las estrategias conllevó un trabajo conjunto antes, durante y después del curso, nutriendo sus prácticas pedagógicas. El DUA en la Educación Superior promueve la transformación de las prácticas docentes, ya que proporciona estrategias específicas para favorecer la motivación y la implicancia de los estudiantes, aspecto esencial para lograr cualquier aprendizaje. Además, ofrece alternativas al profesor para representar de múltiples formas los contenidos, favoreciendo la activación de los diferentes canales sensoriales, y proporciona variedad de estrategias para ofrecer a los estudiantes múltiples formas de expresar y comunicar su aprendizaje, privilegiando sus potencialidades.

Con respecto a la formación de futuros profesores señalar, que la implementación del DUA en el curso herramientas de acceso para el aprendizaje del lenguaje y las matemáticas l, permitió acercar a los estudiantes a su futuro profesional, considerando que la política educativa chilena demanda a los establecimientos educativos, y especialmente a los 
profesores diversificar la enseñanza. Para esto el Ministerio de Educación dispuso el decreto 83, que "se basa en el principio de diseño universal para el aprendizaje que promueve un cambio en el enfoque de la enseñanza, a través de la diversificación de la respuesta educativa [...]" (CHILE, 2017, p. 11).

Es importante señalar algunas limitaciones en la implementación de esta práctica, tales como el espacio físico, específicamente la capacidad de las salas -la que era menor al número de estudiantes matriculados en cada grupo-, y la organización de las horas de clases, pues fueron extensas (cuatro horas continuas). Autores como Gairín (1995) manifiestan que la organización del tiempo y el espacio puede incidir de forma negativa o positiva en el cumplimiento de los objetivos de aprendizaje. En efecto, para implementar estrategias basadas en el DUA se requieren ciertas condiciones mínimas, asociadas a la organización del espacio y el tiempo que faciliten la consecución de las metas de aprendizaje. Por lo tanto, el aula se debe constituir en un lugar en que estos aspectos respondan a las necesidades de aprendizaje de los estudiantes (CELA; PALAU, 1997) y no al servicio de las instituciones educativas (LAORDEN; PÉREZ, 2002). Entre las mejoras que podrían realizarse, se pueden señalar la incorporación del DUA en todos los elementos de currículum levaluación, recursos, métodos y objetivos), así como la elección por parte de los estudiantes de las estrategias y actividades de las clases para favorecer más el interés y la motivación.

Finalmente, esta experiencia nos permite sostener que para crear ambientes de aprendizajes inclusivos desde el DUA es primordial que los profesores seleccionen intencionadamente las pautas y puntos de verificación que utilizarán. Las razones se fundamentan en dos aspectos: i) el DUA es un enfoque que se anticipa a las barreras de aprendizaje y participación; ii) los principios del DUA proporcionan sólo información general, siendo las pautas y puntos de verificación los que proporcionan un mayor nivel de detalle respecto de las estrategias que se podrían implementar en el aula.

\section{REFERENCIAS}

ABELL, M.; JUNG, E.; TAYLOR, M. Students' perceptions of classroom instructional environments in the context of universal design for learning. Learning Environments Research, Australia: Curtin University, v. 14, p. 171-185, 2011.

ALBA, C.; ZUBLLLAGA, A.; SÁNCHEZ, J. Tecnologías y diseño universal para el aprendizaje (DUA): experiencias en el contexto universitario e implicaciones en la formación del profesorado. Revista Latinoamericana de Tecnología Educativa, Cáceres: Universidad de Extremadura, v. 14, n. 1, p. 89-100, 2015.

BARRÍA, C.; GUTIÉRREZ-SALDIVIA, X.; TAPIA, C. (en prensa). Diseño universal para el aprendizaje de las matemáticas en la formación inicial del profesorado. Formación universitaria, La Serena: Centro de Información Tecnológica. 
BROWDER, D. et al. Teaching elementary students with multiple disabilities to participate in shared stories. Research and Practice for Persons with Severe Disabilities, Urbana-Champaign: University of Illinois, v. 33, p. 3-12, 2008.

CELA, J.; PALAU, J. El Espacio. Cuadernos de Pedagogía, Madrid: Wolters Kluwer, v. 254, p. 59-63, 1997.

CENTER FOR APPLIED SPECIAL TECHNOLOGY. Universal design for learning guide-lines version 2.0 . Wakefield, MA: CAST, 2011.

CENTRO DE MODELAMIENTO MATEMÁTICO. Matemática en aula: docentes en acción. Santiago, Chile: Universidad de Chile, 2016.

CHILE. Ministerio de Educación. Decreto $n^{\circ} 83$ Aprueba criterios y orientaciones de adecuación curricular para estudiantes con necesidades educativas especiales de educación parvularia y educación básica. Santiago de Chile: Ministerio de Educación, 2015. Disponible en: http://especial.mineduc.cl/wp-content/uploads/sites/31/2016/08/Decreto-83-2015.pdf. Acceso en: 21 agosto 2019.

CHILE. Ministerio de Educación. Orientaciones sobre estrategias diversificadas de enseñanza para educación básica en el marco del decreto 83/2015. Santiago de Chile: [s. n.], 2017. Disponible en: http:// especial.mineduc.cl/wp-content/uploads/sites/31/2017/05/ORIENTACIONES_D83_Web_05-2017.pdf. Acceso en: 10 dic. 2019.

DEAN, T.; LEE-POST, A.; HAPKE, H. Universal design for leaning large lecture classes. Journal of Marketing Education, Wellesley: Babson College, v. 39, n. 1, p. 5-16, 2016.

DYMOND, S. et al. Using a participatory action research approach to create a universally designed inclusive high school science course: a case study. Research and practice for persons with severe disabilities, Urbana-Champaign: University of Illinois, v. 31, p. 293-308, 2006.

FLORES, D. et al. Las emociones y su impacto en el aprendizaje de las matemáticas. In: CONGRESO IBEROAMERICANO DE EDUCACIÓN MATEMÁTICA, 7., 2013. Montevideo. Anales [...] Montevideo, 2013. p. 23010797.

FREIRE, P. Pedagogía del oprimido. Buenos Aires: Siglo XXI Editores Argentina, 2002.

GAIRÍN, J. El reto de la organización de los espacios. Aula de Innovación Educativa, Barcelona: Graó, v. 39, p. 45-50, 1995.

GUTIÉRREZ-SALDIVIA, X. et al. Acceso a la información en el libro de texto de matemática de primer año en educación primaria en Chile. Espacios, Venezuela: Editorial Espacios Inc, v. 40, n. 42, p. 1-22, 2019. Disponible en: https://www.revistaespacios.com/a19v40n41/a19v40n41p22.pdf. Acceso en: 10 enero 2020. 
GUTIÉRREZ-SALDIVIA, X. Evaluación de la investigación sobre la educación especial en España mediante el análisis cienciométrico de sus tesis doctorales 1978-2013. 2014. Tesis (Magister) - Universidad de Granada, Granada, 2014.

KORTERING, L.; MCCLANNON, T.; BRAZIEL, P. Universal design for learning: a look at what algebra and biology students with and without high incidence conditions are saying. Remedial and Special Education, Lawrence: University of Kansas, v. 29, p. 352-363, 2008.

LIEBER, J. et al. Access to the general education curriculum for preschoolers with disabilities: children's school success. Exceptionality, Gainesville: University of Florida, v. 16, p. 18-32, 2008. D0l: $10.1080 / 09362830701796776$.

LAORDEN, C.; PÉREZ, C. El espacio como elemento facilitador del aprendizaje. Una experiencia en la formación inicial del profesorado. Pulso, Alcalá de Henares: Universidad de Alcalá, v. 25, p. 133-146, 2002.

MANGIATORDI, A.; SERENELLI, F. Universal design for learning: a meta-analytic review of 80 abstracts from peer reviewed journals. Research on Education and Media, Milán: Università Cattolica del Sacro Cuore di Milano, v. 5, n. 1, p. 109-118, 2013.

MEYER, A.; ROSE, D.; GORDON, D. Universal design for learning: theory and practice. Wakefield, MA: CAST, 2014.

PARKER, D.; ROBINSON, L.; HANNAFIN, R. Blending technology and effective pedagogy in a core course for preservice teachers. Journal of Computing in Teacher Education, Ames: Lowa State University, v. 24, p. 49-54, 2008.

RAO, K.; TANNERS, A. Curb cuts in cyberspace: Universal instructional design for online courses. Journal of Postsecondary Education and Disability, Huntersville: Association on Higher Education and Disability, v. 24, p. 211-229, 2011.

RAO, K.; WOOK, M.; BRYANT, B. A review of research on universal design educational models. Remedial and Special Education, Lawrence: University of Kansas, v. 20, n. 10, p. 1-14, 2014.

ROSE, D.; MEYER, A. Teaching every student in the digital age: universal design for learning. Alexandria, VA: Association for Supervision and Curriculum Development, 2002.

SCHELLY, C.; DAVIES, P.; SPOONER, C. Student perceptions of faculty implementation of universal design for learning. Journal of Postsecondary Education and Disability, Huntersville: Association on Higher Education and Disability, v. 24, n. 1, p. 17-30, 2011.

UNIVERSIDAD CATÓLICA DE TEMUCO. Modelo educativo UC Temuco: principios y lineamientos. Temuco, Chile: UCT, 2007. Disponible en: https://uct.cl/archivos/modeloeducativo.pdf. Acceso en: 18 oct. 2019. 
UNIVERSIDAD CATÓLICA DE TEMUCO. Reglamento del estudiante de pregrado de la Universidad Católica de Temuco. Temuco, Chile: UCT, 2018. Disponible en: http://dara.uct.cl/wp-content/uploads/2018/11/1. REGLAMENTO-DE-ESTUDIANTE-REGULAR.pdf. Acceso en: 18 oct. 2019.

YUVAL, L. et al. Evaluation report on the universal instructional design at the University of Guelph. Guelph, ON: University of Guelph, 2004. 
